OPEN ACCESS

Edited by:

Philip Norris,

Blood Systems, United States

Reviewed by:

Soren R. Paludan,

Aarhus University, Denmark

Abel Viejo-Borbolla,

Hannover Medical School, Germany

*Correspondence:

Zheng-Fei Liu

Izf6789@mail.hzau.edu.cn

Specialty section:

This article was submitted to

Viral Immunology,

a section of the journal

Frontiers in Immunology

Received: 14 December 2017

Accepted: 27 March 2018

Published: 12 April 2018

Citation:

Ahmed W and Liu Z-F (2018) Long

Non-Coding RNAs: Novel Players in

Regulation of Immune Response

Upon Herpesvirus Infection.

Front. Immunol. 9:761.

doi: 10.3389/fimmu.2018.00761

\section{Long Non-Coding RNAs: Novel Players in Regulation of Immune Response Upon Herpesvirus Infection}

\author{
Waqas Ahmed ${ }^{1,2}$ and Zheng-Fei Liu' ${ }^{1,3 *}$ \\ ${ }^{1}$ State Key Laboratory of Agricultural Microbiology, College of Veterinary Medicine, Huazhong Agricultural University, Wuhan, \\ China, ${ }^{2}$ College of Life Sciences, Guangzhou University, Guangzhou, China, ${ }^{3}$ Key Laboratory of Preventive Veterinary \\ Medicine in Hubei Province, College of Veterinary Medicine, Huazhong Agricultural University, Wuhan, China
}

Herpesviruses have developed a variety of sophisticated immune evasion strategies to establish lifelong latent infection, including the use of long non-coding RNAs (IncRNAs). In this review, we summarize the IncRNA action modes, i.e., RNA-protein, RNA-RNA, and RNA-DNA interactions, involved in regulating important aspects of immunity by controlling gene expression at various stages. Upon herpesvirus infection, host IncRNAs, such as nuclear paraspeckle assembly transcript 1, negative regulator of antiviral, and B-cell integration cluster have been functionally characterized as negative or positive antiviral regulators in the immune response. Herpesviruses have also evolved multiple strategies to modulate the host immune response using IncRNAs, such as latency-associated transcript, $\beta 2.7$ RNA, 5 kb and 7.2 kb IncRNAs, Epstein-Barr virus-encoded non-coding RNA, BamH I-A rightward transcripts, polyadenylated nuclear, and herpesvirus saimiri U-rich RNAs. We discuss the various mechanisms of immune-related IncRNAs, and their diversified and important functions in the modulation of innate and adaptive immunity upon herpesvirus infection as well as in host-pathogen interactions, which will facilitate our understanding of rational design of novel strategies to combat herpesvirus infection.

Keywords: herpesvirus, long non-coding RNAs, virus infection, innate immunity, adaptive immunity, host-pathogen interaction

\section{INTRODUCTION}

Non-coding RNAs (ncRNAs) play critical roles in cellular functions and are arbitrarily classified as either short ncRNAs ( $<200$ nucleotides) or long ncRNAs ( $>200$ nucleotides) (1-3). Long noncoding RNAs (lncRNAs) are transcribed by RNA polymerase II and do not encode information about proteins. Based on their position relative to protein-coding genes, they are broadly divided into different classes, such as intronic lncRNAs, long intergenic ncRNAs (lincRNAs), antisense lncRNAs, bidirectional lncRNAs, transcribed pseudogene lncRNAs, and enhancer RNAs (eRNAs) (Figure 1A) (4). Thousands of lncRNAs are encoded within the human genome and have been assumed to be "junk" or "dark matter" (5).

The function of lncRNAs has been investigated in a wide range of species, including plants, animals, prokaryotes, and viruses. They were shown to be poorly conserved among species, invoking 


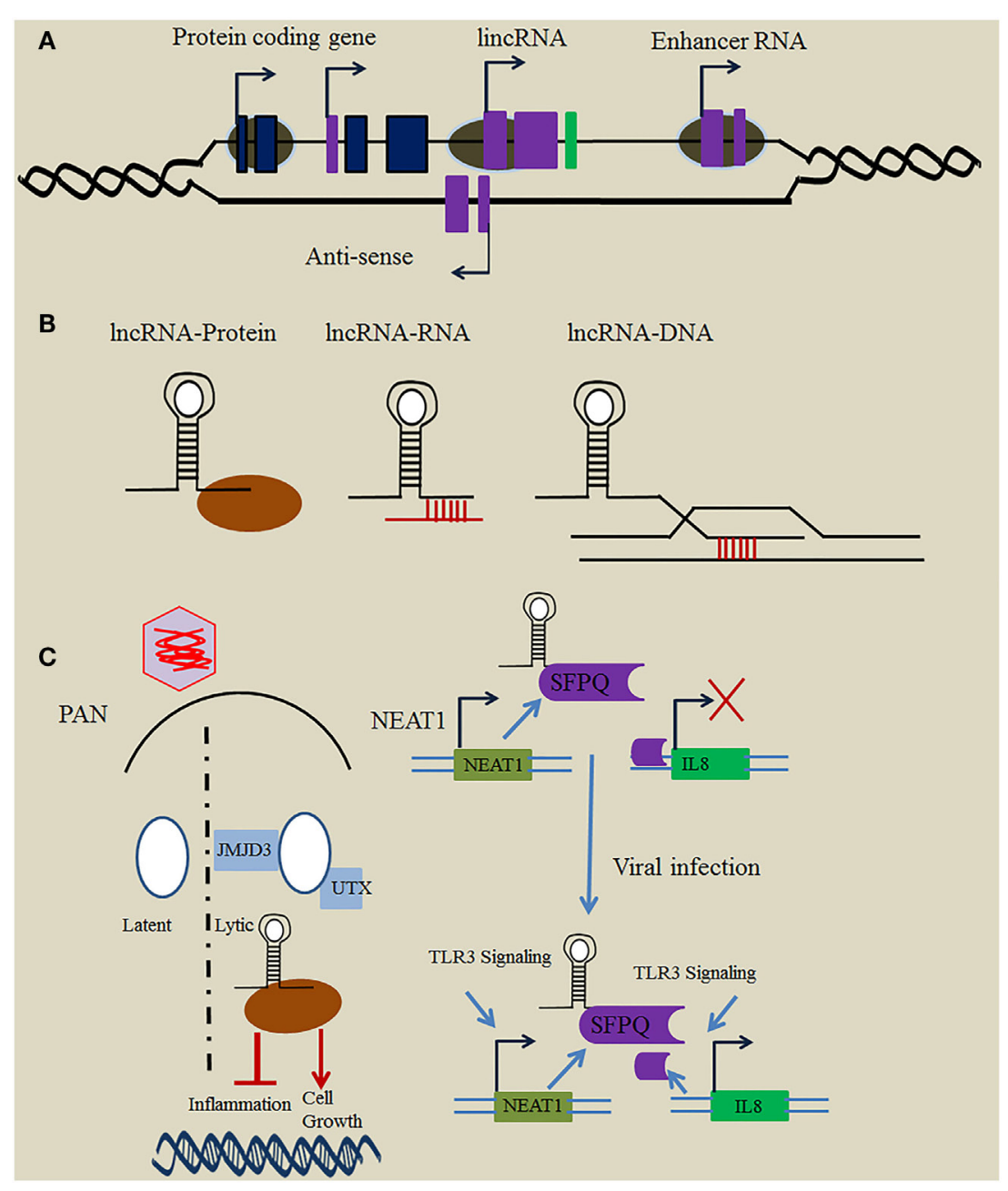

FIGURE 1 | Mechanism employed by long non-coding RNA (IncRNA) in immune regulation. (A) Loci of immune-related IncRNAs relative to protein-coding genes. In general, immune-related IncRNAs are transcribed by RNAP II and classified relative to the position of the neighboring protein-coding gene into different types, including intronic IncRNA, long intergenic ncRNA (lincRNA), enhancer RNA (eRNA), and antisense IncRNA. (B) Mechanisms employed by IncRNAs. IncRNAs use a variety of basic modules to perform their regulatory functions in the cytosol and nucleus through IncRNA-protein, IncRNA-RNA, and IncRNA-DNA interactions. (C) Function of herpesviruses' IncRNAs in immune regulation. The Kaposi's sarcoma-associated herpesvirus (KSHV)-encoded IncRNA polyadenylated nuclear (PAN) binds the histone-modifying complex (demethylases JMJD3 and UTX) which plays an important role in the switch from latent to lytic infection. PAN also subverts the host immune response and modulates viral gene expression through binding with PRC2 to promote cell growth and survival, and to repress the inflammatory response. Nuclear paraspeckle assembly transcript 1 (NEAT1) binds several proteins such as SFPQ, and plays a critical role in regulation of the innate immune response mechanism through the transcriptional regulation of numerous antiviral genes upon herpesvirus infection.

uncertainty about functional species-specific characteristics (6). Growing evidence suggests that lncRNAs contribute to a wide range of biological functions, including cellular structure integrity, splicing, transcription, translation, stem cell pluripotency, the cell cycle, reprogramming, and apoptosis (7-9). lncRNAs were also found to be involved in the development of many human diseases and may regulate cancer progression $(10,11)$. Hence, biological features and functions of lncRNAs make them as an important and interesting research topic.

Herpesviruses are large, enveloped, double-stranded DNA viruses that have infected a wide range of species including animals and humans for hundreds of millions of years. They are classified into three subfamilies: Alphaherpesvirinae, Betaherpesvirinae, and Gammaherpesvirinae $(12,13)$. These viruses can establish lifelong infections in their hosts and are extensive within the human population (14). In immune-compromised individuals, herpesvirus infections result in substantial disease symptoms with a wide range of clinical manifestations spanning from congenital defects, skin/mucosal lesions, and cancer development $(15,16)$.

The ability to establish latency is a major hallmark of herpesvirus infection, and herpesviruses have evolved numerous strategies to evade immune response mechanism to avoid recognition during establishing the latent infection. Recent emerging evidence demonstrates that lncRNAs play key regulatory roles in pathological and physiological responses $(17,18)$. IncRNAs potential function in regulation of immune response mechanism upon herpesvirus infection is now growing and actually the subject that signifies the topic of this review. lncRNAs associated with immune-related 
functions are usually recognized through analysis of differential expression in response to activation of immune cells. The role of specific lncRNAs will be studied under different sections, such as innate immunity, adaptive immunity, and host-pathogen interaction. Nonetheless, understanding the mechanism of action of immune-related lncRNAs will undoubtedly shed more light on their function in immune regulation upon herpesvirus infection.

\section{MECHANISM OF ACTION AND FUNCTION OF IMMUNE-RELATED IncRNAs}

RNA-sequencing analysis revealed that most immune-related lncRNAs partially overlap or are close to the $3^{\prime}$ end (downstream) or $5^{\prime}$ end (upstream) of protein-coding genes and play key roles in the regulation of immune response mechanisms $(19,20)$. The transcription of immune-related lncRNAs usually occurs in the antisense direction, indicating that upstream lncRNAs and mRNAs use a common promoter region to produce bidirectional transcription. Surprisingly, antisense lncRNAs often have no or only a partial overlap with protein-coding genes (21). Many immune-related lncRNAs, including PACER (22), lnc-IL7R (23), THRIL (24), and lincR-Ccr2-50AS (19), have been exposed to have gene regulatory functions in their nearby protein-coding gene in cis.

Although chromatin looping is thought to be essential for placing distal enhancer regions in close proximity with promoter regions, eRNAs have also been reported to show gene regulatory functions in cis. lncRNAs located in intergenic regions, such as HOTAIR, control gene expression to modulate the immune response mechanism in trans (25). Similar to proteins, lncRNAs have modular domains that either enable protein binding through higher order structures or secondary RNA structures by base pairing (Figure 1B) $(1,26)$. Furthermore, lncRNAs also play critical roles as guides, scaffolds, decoys, and signals to regulate a variety of biological processes, including posttranscriptional regulation, transcription, and chromatin remodeling (27). Tumor necrosis factor (TNF) induces the expression of hundreds of lncRNAs in murine fibroblasts. Lethe, a pseudogene lncRNA, is transcribed upon activation of NF- $\mathrm{KB}$, a transcription factor important in inflammation. Lethe binds directly with RelA-RelA homodimers that influence $N F-\kappa B$ response elements, thus hampering the function of downstream effectors, such as NF- $\kappa \mathrm{B}$, interleukin (IL)-6, and IL-8 (28). These findings suggest that Lethe functions as a post-induction feedback regulator of TNF signaling to dampen the inflammatory response. The muscle-specific lncRNA linc-MD1 is expressed during myoblast differentiation, and governs ceRNA to regulate the distribution of miR-135 and miR-133, in turn activating muscle-specific genes $M A M L 1$ and $M E F 2 C$ and regulating myoblast differentiation (29).

\section{IncRNAs IN MODULATION OF INNATE AND ADAPTIVE IMMUNE RESPONSE UPON HERPESVIRUS INFECTION}

Host innate and adaptive immune response mechanisms are comprised of complexes of different biochemical processes regulated by lipid mediators and various proteins, such as pattern recognition receptors, chemokines, cytokines, prostaglandins, hormones, and growth factors. Most lncRNAs were initially thought to be involved in cell differentiation, cancer, and genomic imprinting (30), but growing evidence highlights their function in regulation of both the innate and adaptive immune systems $(31,32)$. Indeed, lncRNAs regulate dendritic cells, macrophages, activator of toll-like receptor 4 (TLR4) signaling, type I interferon (IFN) signaling, T-cell development, and differentiation (33).

Herpesviruses have established well-organized strategies that allow them to exploit and/or evade host immune response mechanisms to persist within its natural host. The function of lncRNAs in immune homeostasis to establish and maintain a herpesvirus latent infection is intriguing, but their biological function in immune regulation is only beginning to emerge. In the following sections, we describe immune-related lncRNAs that play critical roles in modulating the immune response mechanism of herpesviruses through unique mechanisms.

\section{HOST IncRNAs}

Host lncRNAs have been functionally characterized as negative or positive antiviral regulators in the immune response. Interestingly, a number of host lncRNAs can be hijacked and induced by viruses to develop persistent infections, which likely reflects the mutual adaptability of hosts and viruses that has evolved over millions of years. Many non-coding, antisense transcripts have been identified following infection with herpes simplex virus (HSV)-1, with the induction of antisense transcription providing protection against apoptosis. Natural antisense transcripts are involved in regulating gene expression during an immune challenge, suggesting that herpesviruses induce widespread host antisense transcription to interfere with the expression of pro-apoptotic genes (34). Pathogen invasion triggers a number of cellular responses and alters the host transcriptome, as shown for HSV-1 infection which leads to differential gene expression, and changes in RNA splicing and RNA Pol II read-through (35).

\section{NUCLEAR PARASPECKLE ASSEMBLY TRANSCRIPT 1 (NEAT1)}

Nuclear paraspeckle assembly transcript 1 plays an important role in the establishment of nuclear paraspeckles, which are architectural subnuclear component structures formed during the nucleocytoplasmic transport of mRNA in response to stress stimuli (36). It is prompted by HSV infection which leads to the formation of large paraspeckles. NEAT1, also known as virus-inducible ncRNA, binds with several proteins present in paraspeckles such as splicing factor proline and glutamine rich (SFPQ) (37). Imamura et al. (37) explored the function of NEAT1 in the antiviral response within A549 and HeLa cell lines, and demonstrated that NEAT1 accelerates the expression of various antiviral genes such as IL-8. NEAT1 overexpression and knockdown led to the recognition of 85 genes that are directly controlled by NEAT1, including those that play key roles in the antiviral response. SFPQ-NEAT1 binding at the IL- 8 promoter site represses IL-8 transcription; however, higher NEAT1 expression induces the formation of paraspeckles, resulting in transcriptional 
activation of IL8. Furthermore, hexamethylene bis-acetamideinducible protein 1 (HEXIM1) binding with NEAT1 forms a multisubunit complex that regulates the innate immune response through a cGAS-STING-IRF3-dependent pathway. The HEXIM1-DNAPK-paraspeckle components-ribonucleoprotein complex contains various paraspeckle proteins, including MATRIN3, SFPQ, PSPC1, RBM14, and NONO. NEAT1 binding to HEXIM1 was shown to be essential for the formation of this complex (38).

Nuclear paraspeckle assembly transcript 1 upregulated gene expression was observed in Kaposi's sarcoma-associated herpesvirus (KSHV) infection, indicating that KSHV benefits from NEAT1 targeting by preventing the death of KSHV-infected cells (39). In addition, HSV-1 infection leads to NEAT1 upregulated gene expression, resulting in STAT3-dependent paraspeckle formation (40). NONO is a multifunctional DNA-binding protein that is involved in transcriptional regulation of Epstein-Barr virus (EBV). Cao and colleagues reported novel oriP-derived leftward lncRNAs that plays critical function in facilitating viral lytic gene expression, undoubtedly in a manner that initiates the lytic cascade of herpesviruses (41). Taken together, NEAT1 regulates the innate immune response mechanism upon herpesvirus infection by NEAT1 and SFPQ cooperative mechanisms through the transcriptional regulation of numerous antiviral genes (Figure 2).

\section{NEGATIVE REGULATOR OF ANTIVIRAL (NRAV)}

Negative regulator of antiviral is a newly identified lncRNA with an essential function in the regulation of antiviral innate immunity. The downregulation of lncRNA NRAV is assumed to be linked with HSV infection. NRAV facilitates virus replication and significantly downregulates the expression of IFN-stimulated genes (ISGs), including OASL, IFIT2, MxA, IFIT3, and IFITM3 (42). A chief mechanism of lncRNA function is the modular pairing of DNA binding and protein interaction to recruit chromatin-modifying proteins that regulate gene regulation via the chemical modification of histones. NRAV also regulates histone modifications $\mathrm{H} 3 \mathrm{~K} 27 \mathrm{me} 3$ and $\mathrm{H} 3 \mathrm{~K} 4 \mathrm{me} 3$ of ISG genes, which directly inhibits the initial transcription of IFITM3 and MxA (42). $\mathrm{ZO}-1$-associated nucleic acid binding protein is an NRAV-bound protein that acts as a positive regulator; these proteins interact to repress ISG expression in uninfected cells. Taken together, these results indicate that decreases in NRAV might increase the host innate immune defense through the involvement of various antiviral proteins (such as ISGs), thus enabling more efficient viral clearance. NRAV provides good evidence that lncRNAs regulate the antiviral IFN response.

\section{B-CELL INTEGRATION CLUSTER}

The BIC was identified as a non-coding RNA linked with the regulation of numerous aspects of the immune system (43). It is an essential precursor of miR-155, which is highly expressed in activated cells of the immune system (44). BIC is induced by TNF- $\alpha$, IFN- $\beta$, IFN- $\gamma$, TLR signaling, B-cell receptor, and EBV LMP1 and LMP2A engagement (45). Interestingly, the viral miRNA miR-K11, encoded by KSHV, is a functional ortholog of miR-155. EBV was recently shown to diminish NF- $\kappa B$ signaling and suppress host innate immunity to stabilize latent virus

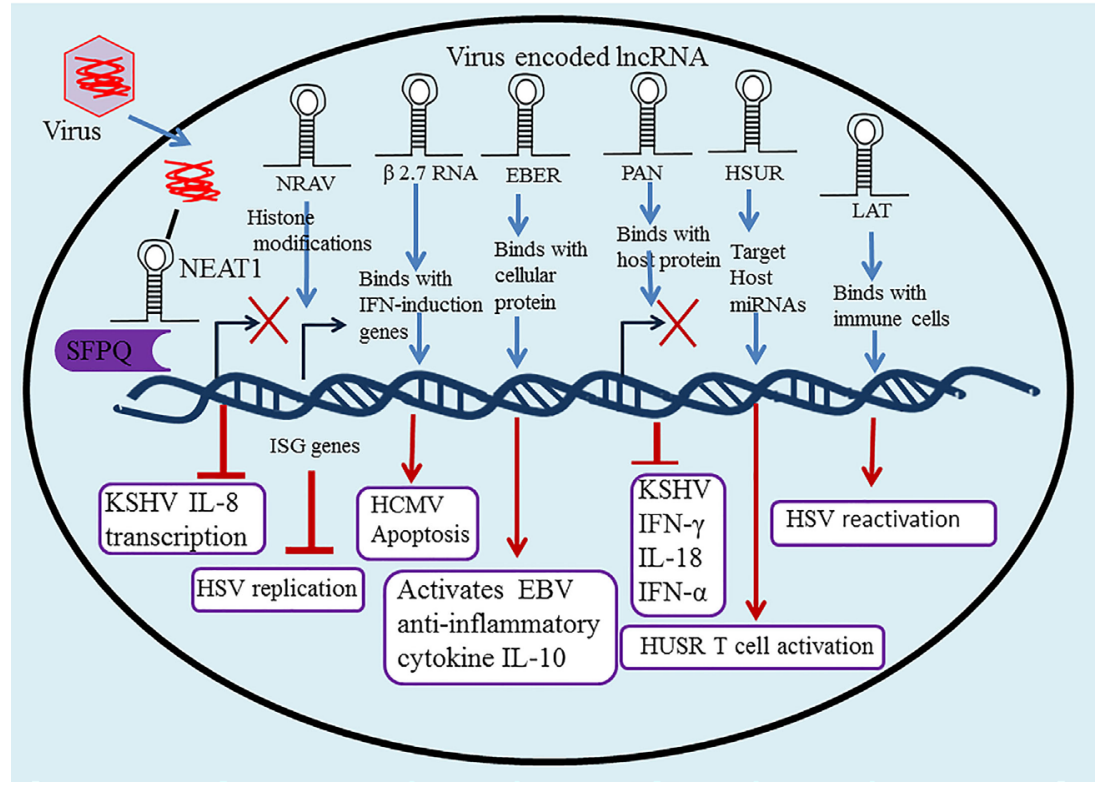

FIGURE 2 | Long non-coding RNA (IncRNA) in regulation of the innate and adaptive immune system upon herpesvirus infection. Many IncRNAs have been associated with regulation of the innate and adaptive immune response and host-pathogen interactions, including nuclear paraspeckle assembly transcript 1 (NEAT1), negative regulator of antiviral (NRAV), polyadenylated nuclear (PAN), Epstein-Barr virus-encoded non-coding RNAs (EBERs), herpesvirus saimiri U-rich RNAs (HSURs), $\beta$ 2.7 RNA, and latency-associated transcript (LAT). 
persistence by inducing miR-155 translational repression (46). Furthermore, BIC has critical functions in lymphomagenesis through the activation of $\mathrm{p} 38 / \mathrm{MAPK}$ and NF- $\mathrm{\kappa B}$ signaling pathways in response to EBV LMP1 (47).

\section{VIRAL IncRNAs}

During viral infections, the host cell responds by producing numerous lncRNAs. Herpesviruses not only alter the expression of immune-related host lncRNAs but also generates their own lncRNAs to modulate the host immune response. In the following section, we describe herpesvirus-encoded lncRNAs that have been identified thus far.

\section{LATENCY-ASSOCIATED TRANSCRIPT (LAT)}

Latency-associated transcript, a non-protein coding RNA that has been strongly implicated in the epigenetic regulation of HSV-1 gene expression, a phenomenon that directly impacts upon the frequency of reactivation and the maintenance of the transcriptionally active latent reservoir (48). LAT constitute a novel immune evasion mechanism whereby the HSV-1 LAT directly or indirectly promotes functional exhaustion (i.e., dysfunction) of HSV-specific CD8+ T cells in latently infected TG, resulting in increased virus reactivation (49). Furthermore, LAT increases reactivation by a direct effect on the reactivation process or by increasing the establishment of latency, thereby making more latently infected neurons available for reactivation (50). HSV-1 LAT contribute to the shaping of a broader repertoire of exhausted $\mathrm{HSV}$-specific CD8+ T cells in latently infected TG via producing less TNF alpha, gamma IFN, and granzyme B, higher levels of PD-1, TIM-3, and CTLA-4 markers of exhaustion, and recognized a broader selection of nonoverlapping HSV-1 epitopes, thus allowing for increased viral reactivation (51).

\section{乃 2.7 RNA}

The 2.7-kb unspliced polyadenylated lncRNA $\beta 2.7$ RNA is the most abundant RNA transcribed in the human cytomegalovirus (HCMV) genome, accounting for some $20 \%$ of all viral RNAs transcript expressed at the early phase of lytic infection (52). Although it possesses coding potential, $\beta$ 2.7 RNA directly binds to genes associated with retinoid/IFN-induced mortality 19 , a subunit of the mitochondrial enzyme complex I. This complex stabilizes the mitochondrial membrane potential which protects virus-infected cells from apoptosis, resulting in continued adenosine triphosphate production for the successful completion of the viral life cycle (53). $\beta$ 2.7 RNA interacts with complex I to prevent rotenone stress-induced apoptosis during both lytic and latent infection in neuronal cells, suggesting a refined strategy by which the virus regulates the metabolic viability of the host cell. Thus, by targeting complex I, $\beta 2.7$ RNA can be used in the development of a novel therapeutic tool (54). $\beta 2.7$ RNA is also a novel effector molecule that reduces the formation of reactive oxygen species by inducing apoptosis, and provides protection to rat aortic endothelial cells from reperfusion/ischemia injury (55).

\section{AND 7.2 kb IncRNAs}

Human cytomegalovirus is a member of Betaherpesvirinae that persistently replicates in glandular epithelial tissue and ultimately develops a lifelong latent infection in the host. HCMV expresses a 5-kb immediate-early stable intronic lncRNA that lacks open reading frames and is highly AT-rich in sequence. It is likely to be translated into protein, so is not required for proliferation of the virus in HCMV-infected cultured fibroblasts (56). A 7.2-kb lncRNA expressed by murine CMV was shown to be an essential element of viral persistence in the salivary gland, facilitating progression from the acute to latent phase of infection $(57,58)$. The CMV intronic lncRNA is extremely long-lived and accumulates in the nuclei of cells during infection, suggesting that stability is a consequence of a sustained lariat conformation $(59,60)$.

\section{EBV-ENCODED NON-CODING RNAS (EBERs)}

EBV-encoded non-coding RNAs EBER1 (167 nt) and EBER2 (172 nt) are two abundant, highly structured, nuclear RNA transcripts produced during latent infection (61). They are nonpolyadenylated, ncRNA transcripts usually transcribed by RNA polymerase III, and involved in regulation of the antiviral innate immune response mechanism through direct interaction with cellular proteins (62). EBERs are recognized by RIG-I, and induce the expression of anti-inflammatory cytokine IL-10 to activate downstream signaling pathways but not NF- $\kappa \mathrm{B}$ activation in EBV-infected cells $(63,64)$. They also play important roles in oncogenesis. For example, they counteract IFN- $\alpha$-induced apoptosis through binding with (ds) RNA-activated protein kinase, resulting in inhibition of its phosphorylation $(65,66)$. EBERs can be identified by their expression of TLR3, which induces inflammatory cytokines and type-I IFNs. Because they are secreted from EBV-infected cells, they can be collected from the sera, suggesting subsequent immune activation using a TLR3-dependent signaling pathway (67).

\section{BamH I-A RIGHTWARD TRANSCRIPTS}

BamH I-A rightward transcripts are non-coding regulatory RNAs that perform identical functions to cellular lncRNAs in the regulation of cellular and/or viral gene expression. Nuclear BART RNAs show increased gene expression which contributes to growth regulation and viral oncogenesis without the expression of immunogenic proteins in EBV-infected cells. This elaborates the generalized mechanism of EBV epithelial latency in the presence of a functional immune system (68). NF- $\kappa B$ prompts the activation of BART promoters and controls the expression of BARTs in EBV-infected cells, as shown by the abolition of BART promoter activity through the introduction of mutations into putative NF- $\kappa \mathrm{B}$ binding sites $(69,70)$. 


\section{POLYADENYLATED NUCLEAR}

The novel abundant 1.2-kb PAN RNA was first investigated in KSHV where it is transcribed by RNA Poly II. PAN RNA plays an important function in controlling viral gene expression and propagation and is involved in subversion of the host immune response $(71,72)$. PAN RNA typically influences cellular and viral gene expression via binding with host proteins, including histone methyltransferase MLL2, histones $\mathrm{H} 1$ and $\mathrm{H} 2 \mathrm{~A}$, the demethylase UTX, poly (A)-binding protein C1, IFN regulatory factor 4 , and polycomb repression complex 2 proteins SUZ12, $\mathrm{EZH} 2$, and JMJD3 $(73,74)$. Its interactions with histonemodifying complexes eliminate the suppressive H3K23me3 mark on the KSHV genome, thus activating lytic replication (Figure 1C). This activation leads to decreased production of viral and inflammatory genes, increased survival, and an enhanced growth phenotype through the epigenetic regulation of KSHV gene expression (75).

Polyadenylated nuclear RNA also decreases the expression of inflammatory cytokines, such as IFN- $\alpha$, IFN- $\gamma$, and IL-18, suggesting it functions as an immune modulator $(76,77)$, while its interaction with the latency-associated nuclear antigen shows it has a role in the maintenance of KSHV latency. The binding of PAN RNA with the viral genome contributes to the switch from latent to lytic infection $(78,79)$. Taken together, these studies demonstrate that viral IncRNA PAN functions as a major global regulator in the regulation of host as well as viral gene expression.

\section{HERPESVIRUS SAIMIRI U-RICH RNAS (HSURs)}

Herpesvirus saimiri U-rich RNAs are viral lncRNAs functioning as miRNA sponges, which cause aggressive lymphoma and T-cell leukemia in latently herpesvirus saimiri (HVS)-infected marmoset $\mathrm{T}$ cells (80). Cazalla et al. (81) documented sequence complementarity between HSUR1 and HSUR2, revealing that target host miRNAs are differently expressed in $\mathrm{T}$ cells. They also showed that HSUR1 and 2 significantly downregulate miR-16 and miR-27, clarifying a viral lncRNA strategy that modulates hostcell gene expression through manipulating the miRNA pathway in a binding dependent, sequence-specific manner (81). A recent study examined the role of miRNAs in HVS-transformed T cells, showing that host miRNAs are directly involved in the downregulation of numerous proteins in the $\mathrm{T}$ cell receptor signaling pathway (80). HSURs 1 and 2 were also reported to upregulate the expression of host genes linked to T cell activation in HVSinfected cells (82). This relationship between host miRNAs and virally encoded lncRNAs may prove to be an important function of viral pathogenesis (83).

\section{CONCLUSION AND FUTURE PERSPECTIVES}

Long non-coding RNAs are fundamental constituents of cells that are considered a major class of RNA genes encoded by the genome, and transcribed and spliced into functional ncRNA molecules. They have been shown to play essential roles in the expression of protein-coding genes, genomic imprinting, genomic activity, cell development, mRNA processing, and dosage compensation, but their precise mechanisms of action still remain unclear. IncRNAs control gene expression in trans or in cis by binding chromatin modifiers and transcription factors, and chromatin remodeling affects their repression or activation. The involvement of lncRNAs in modulation of the immune response mechanism has opened up a new era of immune regulation exploration. IncRNAs participate in numerous biological mechanisms of antiviral innate immunity. They are differentially expressed as positive or negative regulators during several important stages of antiviral response, including the expression of virus-induced cytokines, chemokines, and IFNs, the activation of JAK-STAT signaling, host PRR signaling-activated TFs, and ISG transcription. Interestingly, the activity and altered expression of lncRNAs considerably affect the host antiviral immune response mechanism, thereby interrupting viral replication and infection.

Recent studies have provided strong evidence supporting the functions of lncRNAs in the modulation of innate and adaptive herpesvirus immune response mechanisms as well as in hostpathogen interactions. Although several lncRNAs are associated with viral infection, their involvement and experimental validation in herpesvirus infection is very limited. However, research into lncRNA roles in immunity is still in its infancy. This review summarizes the functions of individual lncRNAs in immune responses and IncRNA-mediated regulation of host-pathogen interactions. However, additional studies are required to explore the function, gene expression regulation, and involvement of lncRNAs in viral pathogenesis and immune regulation.

It is worth mentioning particular research topics that should be addressed in the field of immune-regulatory lncRNAs in the future. Functional crosstalk between lncRNAs and miRNAs in the immune regulation of herpesviruses is an important area of interest. Second, defining the key roles of immune-related lncRNAs in vivo using animal models will aid understanding of the functions of lncRNAs in the immune response. Finally, lncRNAs are thought to be translated and coded as peptides or small proteins, so it would be beneficial to determine their coding potentials and the roles of coding peptides in immune regulation. A better understanding of the biology of $\operatorname{lncRNAs}$ will facilitate our comprehension of their functional mechanisms, and pave the way for the prophylaxis and treatment of many infectious diseases, including herpesvirus infections.

\section{AUTHOR CONTRIBUTIONS}

Z-FL conceived the research. WA and Z-FL wrote the manuscript. All authors have read and approved the manuscript.

\section{FUNDING}

This work was supported by the Natural Science Foundation of China $(31470259,31770191)$ and the National Key Research and Development Program (2016YFD0500105) to Z-FL. 


\section{REFERENCES}

1. Guttman M, Rinn JL. Modular regulatory principles of large non-coding RNAs. Nature (2012) 482:339-46. doi:10.1038/nature10887

2. Hu WQ, Alvarez-Dominguez JR, Lodish HF. Regulation of mammalian cell differentiation by long non-coding RNAs. EMBO Rep (2012) 13:971-83. doi:10.1038/embor.2012.145

3. Ulitsky I, Bartel DP. lincRNAs: genomics, evolution, and mechanisms. Cell (2013) 154:26-46. doi:10.1016/j.cell.2013.06.020

4. Dykes IM, Emanueli C. Transcriptional and post-transcriptional gene regulation by long non-coding RNA. Genomics Proteomics Bioinform (2017) 15:177-86. doi:10.1016/j.gpb.2016.12.005

5. Doolittle WF. Is junk DNA bunk? A critique of ENCODE. Proc Natl Acad Sci US A (2013) 110:5294-300. doi:10.1073/pnas.1221376110

6. Ma LN, Bajic VB, Zhang Z. On the classification of long non-coding RNAs. RNA Biol (2013) 10:925-34. doi:10.4161/rna.24604

7. Ponting CP, Oliver PL, Reik W. Evolution and functions of long noncoding RNAs. Cell (2009) 136:629-41. doi:10.1016/j.cell.2009.02.006

8. Clark MB, Mattick JS. Long noncoding RNAs in cell biology. Semin Cell Dev Biol (2011) 22:366-76. doi:10.1016/j.semcdb.2011.01.001

9. Xu SS, Dong LL, Shi YY, Chen LJ, Yuan PP, Wang S, et al. The novel landscape of long non-coding RNAs in response to human foamy virus infection characterized by RNA-Seq. AIDS Res Hum Retroviruses (2017) 33:452-64. doi:10.1089/aid.2016.0156

10. Wapinski O, Chang HY. Long noncoding RNAs and human disease. Trends Cell Biol (2011) 21:354-61. doi:10.1016/j.tcb.2011.04.001

11. Kwok ZH, Tay Y. Long noncoding RNAs: lincs between human health and disease. Biochem Soc Trans (2017) 45:805-12. doi:10.1042/Bst20160376

12. Davison AJ, Eberle R, Ehlers B, Hayward GS, McGeoch DJ, Minson AC, et al. The order herpesvirales. Arch Virol (2009) 154:171-7. doi:10.1007/ s00705-008-0278-4

13. Dittmer DP, Damania B. Kaposi sarcoma associated herpesvirus pathogenesis (KSHV) - an update. Curr Opin Virol (2013) 3:238-44. doi:10.1016/j. coviro.2013.05.012

14. McGeoch DJ, Gatherer D. Integrating reptilian herpesviruses into the family herpesviridae. J Virol (2005) 79:725-31. doi:10.1128/Jvi.79.2.725-731.2005

15. Zamora MR. DNA viruses (CMV, EBV, and the herpesviruses). Semin Respir Crit Care Med (2011) 32:454-70. doi:10.1055/s-0031-1283285

16. Li Z, Fu SJ, Sun LQ. Viral noncoding RNAs in cancer biology. Adv Exp Med Biol (2016) 927:367-89. doi:10.1007/978-981-10-1498-7_14

17. Geisler S, Coller J. RNA in unexpected places: long non-coding RNA functions in diverse cellular contexts. Nat Rev Mol Cell Biol (2013) 14:699-712. doi:10.1038/nrm3679

18. Quan MY, Chen JH, Zhang DQ. Exploring the secrets of long noncoding RNAs. Int J Mol Sci (2015) 16:5467-96. doi:10.3390/ijms16035467

19. Hu GQ, Tang QS, Sharma S, Yu F, Escobar TM, Muljo SA, et al. Expression and regulation of intergenic long noncoding RNAs during $\mathrm{T}$ cell development and differentiation. Nat Immunol (2013) 14:1190-U1118. doi:10.1038/ni.2712

20. Deniz E, Erman B. Long noncoding RNA (lincRNA), a new paradigm in gene expression control. Funct Integr Genomics (2017) 17:135-43. doi:10.1007/ s10142-016-0524-X

21. Bonasio R, Shiekhattar R. Regulation of transcription bylong noncoding RNAs. Annu Rev Genet (2014) 48:433-55. doi:10.1146/annurev-genet-120213-092323

22. Krawczyk M, Emerson BM. p50-Associated COX-2 extragenic RNA (PACER) activates COX-2 gene expression by occluding repressive NF-kappa B complexes. Elife (2014) 3:e01776. doi:10.7554/eLife.01776

23. Cui HC, Xie N, Tan Z, Banerjee S, Thannickal VJ, Abraham E, et al. The human long noncoding RNA lnc-IL7R regulates the inflammatory response. Eur J Immunol (2014) 44:2085-95. doi:10.1002/eji.201344126

24. Li ZH, Chao TC, Chang KY, Lin NW, Patil VS, Shimizu C, et al. The long noncoding RNA THRIL regulates TNF alpha expression through its interaction with hnRNPL. Proc Natl Acad Sci U S A (2014) 111:1002-7. doi:10.1073/ pnas.1313768111

25. Gupta RA, Shah N, Wang KC, Kim J, Horlings HM, Wong DJ, et al. Long non-coding RNA HOTAIR reprograms chromatin state to promote cancer metastasis. Nature (2010) 464:1071-6. doi:10.1038/nature08975

26. Mercer TR, Dinger ME, Mattick JS. Long non-coding RNAs: insights into functions. Nat Rev Genet (2009) 10:155-9. doi:10.1038/nrg2521
27. Yoon JH, Abdelmohsen K, Gorospe M. Posttranscriptional gene regulation by long noncoding RNA. J Mol Biol (2013) 425:3723-30. doi:10.1016/j. jmb.2012.11.024

28. Rapicavoli NA, Qu K, Zhang JJ, Mikhail M, Laberge RM, Chang HY. A mammalian pseudogene lncRNA at the interface of inflammation and anti-inflammatory therapeutics. Elife (2013) 2:e00762. doi:10.7554/ eLife.00762

29. Cesana M, Cacchiarelli D, Legnini I, Santini T, Sthandier O, Chinappi M, et al. A long noncoding RNA controls muscle differentiation by functioning as a competing endogenous RNA (vol 147, pg 358, 2011). Cell (2011) 147:947. doi:10.1016/j.cell.2011.10.031

30. Atianand MK, Fitzgerald KA. Long non-coding RNAs and control of gene expression in the immune system. Trends Mol Med (2014) 20:623-31. doi:10.1016/j.molmed.2014.09.002

31. Fitzgerald KA, Caffrey DR. Long noncoding RNAs in innate and adaptive immunity. Curr Opin Immunol (2014) 26:140-6. doi:10.1016/j.coi.2013.12.001

32. Zhang Y, Cao XT. Long noncoding RNAs in innate immunity. Cell Mol Immunol (2016) 13:138-47. doi:10.1038/cmi.2015.68

33. Heward JA, Lindsay MA. Long non-coding RNAs in the regulation of the immune response. Trends Immunol (2014) 35:408-19. doi:10.1016/j. it.2014.07.005

34. Wyler E, Menegatti J, Franke V, Kocks C, Boltengagen A, Hennig T, et al. Widespread activation of antisense transcription of the host genome during herpes simplex virus 1 infection. Genome Biol (2017) 18:209. doi:10.1186/ s13059-017-1329-5

35. Hu B, Li X, Huo Y, Yu Y, Zhang Q, Chen G, et al. Cellular responses to HSV-1 infection are linked to specific types of alterations in the host transcriptome. Sci Rep (2016) 6:28075. doi:10.1038/srep28075

36. Naganuma T, Hirose T. Paraspeckle formation during the biogenesis of long non-coding RNAs. RNA Biol (2013) 10:456-61. doi:10.4161/rna.23547

37. Imamura K, Imamachi N, Akizuki G, Kumakura M, Kawaguchi A, Nagata $\mathrm{K}$, et al. Long noncoding RNA NEAT1-dependent SFPQ relocation from promoter region to paraspeckle mediates IL8 expression upon immune stimuli (vol 53, pg 393, 2014). Mol Cell (2014) 54:1055. doi:10.1016/j. molcel.2014.06.013

38. Morchikh M, Cribier A, Raffel R, Amraoui S, Cau JL, Severac D, et al. HEXIM1 and NEAT1 long non-coding RNA form a multi-subunit complex that regulates DNA-mediated innate immune response. Mol Cell (2017) 67:387-99.e5. doi:10.1016/j.molcel.2017.06.020

39. Viollet C, Davis DA, Tekeste SS, Reczko M, Ziegelbauer JM, Pezzella F, et al. RNA sequencing reveals that Kaposi sarcoma-associated herpesvirus infection mimics hypoxia gene expression signature. PLoS Pathog (2017) 13:e1006143. doi:10.1371/journal.ppat.1006143

40. Wang ZQ, Fan P, Zhao YW, Zhang SK, Lu JH, Xie WD, et al. NEAT1 modulates herpes simplex virus-1 replication by regulating viral gene transcription. Cell Mol Life Sci (2017) 74:1117-31. doi:10.1007/s00018-016-2398-4

41. Cao SB, Moss W, O’Grady T, Concha M, Strong MJ, Wang X, et al. New noncoding lytic transcripts derived from the Epstein-Barr virus latency origin of replication, oriP, are hyperedited, bind the paraspeckle protein, NONO/ p54nrb, and support viral lytic transcription. J Virol (2015) 89:7120-32. doi:10.1128/Jvi.00608-15

42. Ouyang J, Hu JY, Chen JL. IncRNAs regulate the innate immune response to viral infection. Wiley Interdiscip Rev RNA (2016) 7:129-43. doi:10.1002/ wrna. 1321

43. Tsai CY, Allie SR, Zhang WJ, Usherwood EJ. MicroRNA miR-155 affects antiviral effector and effector memory CD8 T cell differentiation. J Virol (2013) 87:2348-51. doi:10.1128/Jvi.01742-12

44. O'Connell RM, Taganov KD, Boldin MP, Cheng GH, Baltimore D. MicroRNA-155 is induced during the macrophage inflammatory response. Proc Natl Acad Sci U S A (2007) 104:1604-9. doi:10.1073/pnas.0610731104

45. Moynagh PN. TLR signalling and activation of IRFs: revisiting old friends from the NF-kappa B pathway. Trends Immunol (2005) 26:469-76. doi:10.1016/ j.it.2005.06.009

46. Lu F, Weidmer A, Liu CG, Volinia S, Croce CM, Lieberman PM. Epstein-Barr virus-induced miR-155 attenuates NF-kappa B signaling and stabilizes latent virus persistence. J Virol (2008) 82:10436-43. doi:10.1128/Jvi.00752-08

47. Rahadiani N, Takakuwa T, Tresnasari K, Morii E, Aozasa K. Latent membrane protein-1 of Epstein-Barr virus induces the expression of B-cell integration 
cluster, a precursor form of microRNA-155, in B lymphoma cell lines. Biochem Biophys Res Commun (2008) 377:579-83. doi:10.1016/j.bbrc.2008.10.007

48. Nicoll MP, Hann W, Shivkumar M, Harman LE, Connor V, Coleman HM, et al. The HSV-1 latency-associated transcript functions to repress latent phase lytic gene expression and suppress virus reactivation from latently infected neurons. PLoS Pathog (2016) 12:e1005539. doi:10.1371/journal.ppat.1005539

49. Srivastava R, Khan AA, Huang J, Nesburn AB, Wechsler SL, BenMohamed L. A herpes simplex virus type 1 human asymptomatic CD8+ T-cell epitopes-based vaccine protects against ocular herpes in a "humanized" HLA transgenic rabbit model. Invest Ophthalmol Vis Sci (2015) 56:4013-28. doi:10.1167/iovs.1517074

50. Perng G-C, Slanina SM, Yukht A, Ghiasi H, Nesburn AB, Wechsler SL. The latency-associated transcript gene enhances establishment of herpes simplex virus type 1 latency in rabbits. J Virol (2000) 74:1885-91. doi:10.1128/ JVI.74.4.1885-1891.2000

51. Srivastava R, Dervillez X, Khan AA, Chentoufi AA, Chilukuri S, Shukr N, et al. The herpes simplex virus latency-associated transcript gene is associated with a broader repertoire of virus-specific exhausted CD8+ T cells retained within the trigeminal ganglia of latently infected HLA transgenic rabbits. J Virol (2016) 90:3913-28. doi:10.1128/JVI.02450-15

52. Gatherer D, Seirafian S, Cunningham C, Holton M, Dargan DJ, Baluchova K, et al. High-resolution human cytomegalovirus transcriptome. Proc Natl Acad Sci U S A (2011) 108:19755-60. doi:10.1073/pnas.1115861108

53. Reeves MB, Davies AA, McSharry BP, Wilkinson GW, Sinclair JH. Complex I binding by a virally encoded RNA regulates mitochondria-induced cell death. Science (2007) 316:1345-8. doi:10.1126/science.1142984

54. Poole E, Kuan WL, Barker R, Sinclair J. The human cytomegalovirus non-coding Beta2.7 RNA as a novel therapeutic for Parkinson's disease - translational research with no translation. Virus Res (2016) 212:64-9. doi:10.1016/j. virusres.2015.05.007

55. Zhao J, Sinclair J, Houghton J, Bolton E, Bradley A, Lever A. Cytomegalovirus beta 2.7 RNA transcript protects endothelial cells against apoptosis during ischemia/reperfusion injury. JHeart Lung Transplant (2010) 29:342-5. doi:10.1016/j.healun.2009.09.006

56. Kulesza CA, Shenk T. Human cytomegalovirus 5-kilobase immediate-early RNA is a stable intron. J Virol (2004) 78:13182-9. doi:10.1128/ Jvi.78.23.13182-13189.2004

57. Kulesza CA, Shenk T. Murine cytomegalovirus encodes a stable intron that facilitates persistent replication in the mouse. Proc Natl Acad Sci U S A (2006) 103:18302-7. doi:10.1073/pnas.0608718103

58. Schwarz TM, Kulesza CA. Stability determinants of murine cytomegalovirus long noncoding RNA7.2. J Virol (2014) 88:11630-3. doi:10.1128/Jvi.01695-14

59. Lisnic VJ, Cac MB, Lisnic B, Trsan T, Mefferd A, Das Mukhopadhyay C, et al. Dual analysis of the murine cytomegalovirus and host cell transcriptomes reveal new aspects of the virus-host cell interface. PLoS Pathog (2013) 9:e1003611. doi:10.1371/journal.ppat.1003611

60. Schwarz TM, Volpe LAM, Abraham CG, Kulesza CA. Molecular investigation of the $7.2 \mathrm{~kb}$ RNA of murine cytomegalovirus. Virol $J$ (2013) 10:348. doi:10.1186/1743-422x-10-348

61. Iwakiri D. Multifunctional non-coding Epstein-Barr virus encoded RNAs (EBERs) contribute to viral pathogenesis. Virus Res (2016) 212:30-8. doi:10.1016/j.virusres.2015.08.007

62. Samanta M, Iwakiri D, Takada K. Epstein-Barr virus-encoded small RNA induces IL-10 through RIG-I-mediated IRF-3 signaling. Oncogene (2008) 27:4150-60. doi:10.1038/onc.2008.75

63. Samanta M, Iwakiri D, Kanda T, Imaizumi T, Takada K. EB virus-encoded RNAs are recognized by RIG-I and activate signaling to induce type IIFN. EMBO J (2006) 25:4207-14. doi:10.1038/sj.emboj.7601314

64. Iwakiri D, Takada K. Role of EBERs in the pathogenesis of EBV infection. Adv Cancer Res (2010) 107:119-36. doi:10.1016/S0065-230x(10)07004-1

65. Nanbo A, Inoue K, Adachi-Takasawa K, Takada K. Epstein-Barr virus RNA confers resistance to interferon-alpha-induced apoptosis in Burkitt's lymphoma. EMBO J (2002) 21:954-65. doi:10.1093/emboj/21.5.954

66. Nanbo A, Takada K. The role of Epstein-Barr virus-encoded small RNAs (EBERs) in oncogenesis. Rev Med Virol (2002) 12:321-6. doi:10.1002/rmv.363

67. Nanbo A, Yoshiyama H, Nanbo A. Epstein-Barr virus-encoded poly(A)(-) RNA confers resistance to apoptosis mediated through Fas by blocking the
PKR pathway in human epithelial intestine 407 cells. J Virol (2005) 79:12280-5. doi:10.1128/Jvi.79.19.12280-12285.2005

68. Marquitz AR, Mathur A, Edwards RH, Raab-Traub N. Host gene expression is regulated by two types of noncoding RNAs transcribed from the EpsteinBarr virus BamHI A rightward transcript region. J Virol (2015) 89:11256-68. doi:10.1128/Jvi.01492-15

69. Skalsky RL, Cullen BR. EBV noncoding RNAs. Curr Top Microbiol Immunol (2015) 391:181-217. doi:10.1007/978-3-319-22834-1_6

70. Verhoeven RJA, Tong S, Zhang GH, Zong JF, Chen YX, Jin DY, et al. NF-kappa B signaling regulates expression of Epstein-Barr virus BART microRNAs and long noncoding RNAs in nasopharyngeal carcinoma. JVirol (2016) 90:6475-88. doi:10.1128/Jvi.00613-16

71. Rossetto CC, Pari GS. Kaposi's sarcoma-associated herpesvirus noncoding polyadenylated nuclear RNA interacts with virus- and host cell-encoded proteins and suppresses expression of genes involved in immune modulation. J Virol (2011) 85:13290-7. doi:10.1128/Jvi.05886-11

72. Conrad NK. New insights into the expression and functions of the Kaposi's sarcoma-associated herpesvirus long noncoding PAN RNA. Virus Res (2016) 212:53-63. doi:10.1016/j.virusres.2015.06.012

73. Rossetto CC, Tarrant-Elorza M, Verma S, Purushothaman P, Pari GS. Regulation of viral and cellular gene expression by Kaposi's sarcoma-associated herpesvirus polyadenylated nuclear RNA. J Virol (2013) 87:5540-53. doi:10.1128/Jvi.03111-12

74. Rossetto CC, Tarrant-Elorza M, Verma S, Purushothaman P, Pari GS. Regulation of viral and cellular gene expression by Kaposi's sarcoma-associated herpesvirus polyadenylated nuclear RNA (vol 87, pg 5540, 2013). J Virol (2016) 90:4255. doi:10.1128/JVI.00171-16

75. Rossetto CC, Pari G. KSHV PAN RNA associates with demethylases UTX and JMJD3 to activate lytic replication through a physical interaction with the virus genome. PLoS Pathog (2012) 8:e1002680. doi:10.1371/journal. ppat. 1002680

76. Borah S, Darricarrere N, Darnell A, Myoung J, Steitz JA. A viral nuclear noncoding RNA binds re-localized Poly(A) binding protein and is required for late KSHV gene expression. PLoS Pathog (2011) 7:e1002300. doi:10.1371/ journal.ppat. 1002300

77. Rossetto CC, Pari GS. PAN's labyrinth: molecular biology of Kaposi's sarcoma-associated herpesvirus (KSHV) PAN RNA, a multifunctional long noncoding RNA. Viruses (2014) 6:4212-26. doi:10.3390/v6114212

78. Campbell M, Kim KY, Chang PC, Huerta S, Shevchenko B, Wang DH, et al. A lytic viral long noncoding RNA modulates the function of a latent protein. J Virol (2014) 88:1843-8. doi:10.1128/Jvi.03251-13

79. Uppal T, Banerjee S, Sun ZG, Verma SC, Robertson ES. KSHV LANA-the master regulator of KSHV latency. Viruses (2014) 6:4961-98. doi:10.3390/ v6124961

80. Guo YE, Oei T, Steitz JA. Herpesvirus saimiri microRNAs preferentially target host cell cycle regulators. J Virol (2015) 89:10901-11. doi:10.1128/Jvi.01884-15

81. Cazalla D, Yario T, Steitz J. Down-regulation of a host microRNA by a herpesvirus saimiri noncoding RNA. Science (2010) 328:1563-6. doi:10.1126/ science. 1187197

82. Cook HL, Lytle JR, Mischo HE, Li MJ, Rossi JJ, Siva DP, et al. Small nuclear RNAs encoded by herpesvirus saimiri upregulate the expression of genes linked to T cell activation in virally transformed T cells. Curr Biol (2005) 15:974-9. doi:10.1016/j.cub.2005.04.034

83. Tavanez JP, Quina AS, Cunha C. Virus and noncoding RNAs: stars in the host-virus interaction game. Future Virol (2014) 9:1077-87. doi:10.2217/ Fvl.14.84

Conflict of Interest Statement: The authors declare that the research was conducted in the absence of any commercial or financial relationships that could be construed as a potential conflict of interest.

Copyright $\odot 2018$ Ahmed and Liu. This is an open-access article distributed under the terms of the Creative Commons Attribution License (CC BY). The use, distribution or reproduction in other forums is permitted, provided the original author(s) and the copyright owner are credited and that the original publication in this journal is cited, in accordance with accepted academic practice. No use, distribution or reproduction is permitted which does not comply with these terms. 